Editorial

\title{
Special Issue on Advanced DSP Techniques for High-Capacity and Energy-Efficient Optical Fiber Communications
}

\author{
Zhongqi Pan ${ }^{1}\left[\right.$ and Yang Yue ${ }^{2, *}$ \\ 1 Department of Electrical and Computer Engineering, University of Louisiana at Lafayette, \\ Lafayette, LA 70504, USA; zpan@louisiana.edu \\ 2 Institute of Modern Optics, Nankai University, Tianjin 300350, China \\ * Correspondence: yueyang@nankai.edu.cn; Tel.: +86-22-8535-8565
}

Received: 8 October 2019; Accepted: 18 October 2019; Published: 22 October 2019

\section{Introduction}

The rapid proliferation of the Internet has been driving communication networks closer and closer to their limits, while available bandwidth is disappearing due to ever-increasing network loads. In the past decade, optical fiber communication technology has increased the per fiber data rate from $10 \mathrm{~Tb} / \mathrm{s}$ to over $10 \mathrm{~Pb} / \mathrm{s}$ [1]. A major explosion came after the maturity of coherent detection and advanced digital signal processing (DSP), which enabled the achievement of high spectral and energy efficiency. It is difficult to overstate the impact that optical coherent technologies have had in both generating and supporting the revolution of optical communications over the last 10 years.

As one of the key enablers in the coherent evolution of fiber communication systems, DSP has made the innovation of high-order modulation formats possible in increasing spectral/power efficiency. DSP can also compensate almost all linear and nonlinear distortions, and improve noise tolerance in fiber systems. It provides a promising electrical solution for many problems in the optical domain. For example, troublesome channel impairments due to chromatic dispersion (CD) and polarization mode dispersion (PMD) can be almost fully compensated through DSP-based equalizers. Furthermore, DSP has the potential to ease the requirements for many optical components and can even take the place of some optical functions.

\section{Special Issue Papers}

In this Special Issue, we present nine carefully selected papers, including three review articles and six contributed papers. These papers cover advanced DSP techniques for long-distance, short-reach applications, including systems that use conventional single mode fibers (SMFs) and those based on space-division multiplexing (SDM) fibers, as well as links that use free-space wireless transmission.

The following summarizes these papers:

- Advanced DSP for Coherent Optical Fiber Communication [2]

This paper provides an overview of recent progress on advanced DSP techniques for high-capacity, long-haul, coherent optical fiber transmission systems. The authors first introduce the principle and scheme of coherent detection to explain why DSP can compensate for transmission impairments. Then, the corresponding techniques for nonlinearity compensation, frequency-domain equalization (FDE), SDM, and machine learning (ML) are discussed. Relevant techniques are analyzed, and representational results and experimental verifications are demonstrated as well. This paper also provides a brief conclusion and future perspectives at the end. 
- $\quad$ Recent Advances in DSP Techniques for Mode Division Multiplexing Optical Networks with MIMO Equalization: A Review [3]

This paper provides a technical review regarding the latest progress on multi-input multi-output (MIMO) DSP equalization techniques for high-capacity fiber-optic communication networks. The authors discuss state of the art of MIMO equalizers, predominantly focusing on the advantages of implementing the space-time block coding (STBC)-assisted MIMO technique. They also present a performance evaluation of different MIMO frequency-domain equalization (FDE) schemes for differential mode group delay (DMGD) and mode-dependent loss (MDL) issues in adaptive coherent receivers. Moreover, optimization of hardware complexity in MIMO-DSP is discussed, and a joint-compensation scheme is deliberated for CD and DMGD, along with a number of recent experimental demonstrations using MIMO-DSP.

- Recent Advances in Equalization Technologies for Short-Reach Optical Links Based on PAM4 Modulation: A Review [4]

The authors review the latest progress on DSP equalization technologies for short-reach optical links based on four-level pulse amplitude modulation (PAM4) modulation. They introduce the configuration and challenges of the transmission system, and cover the principles and performance of different equalizers and some improved methods. In addition, machine learning algorithms are discussed to mitigate nonlinear distortion for next-generation short-reach PAM4 links. A summary of various equalization technologies is illustrated, and a perspective of the future trend is given as well.

- Experimental Investigation of $400 \mathrm{~Gb} / \mathrm{s}$ Data Center Interconnect Using Unamplified High-Baud-Rate and High-Order QAM Single-Carrier Signal [5]

The authors review the latest progress on data center interconnects (DCI). They also discuss different perspectives on the 400G pluggable module, including form factor, architecture, digital signal processing (DSP), and module power consumption, following 400G pluggable optics in DCI applications. The authors also experimentally investigate the capacity-reach matrix for high-baud-rate and high-order quadrature amplitude modulation (QAM) single-carrier signals in unamplified single-mode optical fiber (SMF) links.

- Compensation of Limited Bandwidth and Nonlinearity for Coherent Transponder [6]

The authors present a novel solution for optimizing the coefficients of digital filters to mitigate impairments due to limited bandwidth and nonlinearity in coherent transponders. They show that limited bandwidth is improved by the finite impulse response filter, while nonlinearity is mitigated by the memoryless Volterra filter.

- Optical Transmitters without Driver Amplifiers-Optimal Operation Conditions [7]

The authors discuss the influence of waveform design on the root-mean-square amplitude and the optical signal quality generated by a Mach-Zehnder modulator with a limited electrical swing (Vpp). Specifically, the influence of the pulse shape, clipping, and digital pre-distortion on the signal quality after the electrical-to-optical conversion are investigated. The findings are of interest for single-channel intensity modulation and direct detection (IM/DD) links, as well as optical coherent communication links.

- Performance Improvement for Mixed RF-FSO Communication System by Adopting Hybrid Subcarrier Intensity Modulation [8]

This paper presents research on end-to-end mixed radio frequency-free space optical (RF-FSO) systems with the hybrid pulse position modulation-binary phase shift keying-subcarrier intensity modulation (PPM-BPSK-SIM) scheme in wireless optical communications. The RF link obeys Rayleigh distribution and the FSO link experiences gamma-gamma distribution. The average bit error rate (BER) for various PPM-BPSK-SIM schemes is derived with consideration of atmospheric 
turbulence influence and pointing error condition. The outage probability and the average channel capacity of the system are discussed as well.

- $\quad$ Post-FEC Performance of Pilot-Aided Carrier Phase Estimation over Cycle Slip [9]

The authors present the post-forward error correction (FEC) bit error rate (BER) performance and the cycle-slip (CS) probability of the carrier phase estimation (CPE) scheme based on the Viterbi-Viterbi phase estimation (VVPE) algorithm and the VV cascaded by the pilot-aided-phase-unwrap (PAPU) algorithm in a 56 Gbit/s quadrature phase-shift keying (QPSK) coherent communication system.

- IBP Based Caching Strategy in D2D [10]

Device-to-device (D2D) communication is a key technology in 5G wireless systems, increasing communication capacity and spectral efficiency. In this paper, the authors propose an Indian buffet process-based D2D caching strategy (IBPSC) to provide high quality D2D communications according to physical closeness between devices. Experimental results show that IBPSC achieves the best network performance.

\section{Future Trend}

Network traffic has been increasing exponentially over decades. This enormous growth rate will continue, in the foreseeable future, due to many newly-emerging and unanticipated digital applications and services in the 5G network. To fulfill the ever-growing bandwidth demand, not only do the spectral efficiencies of optical fiber communication systems need to be further improved, but also the power/wavelength needs to be reduced so that higher individual data rates per wavelength (up to multi- $\mathrm{Tb} / \mathrm{s}$ ) can be achieved with total aggregate capacities well beyond $\mathrm{Pb} / \mathrm{s}$. As one of the most prominent enabling technologies, DSP has played a critical role in accommodating channel impairment mitigation, enabling advanced modulation formats for spectral-efficient transmission, and realizing flexible bandwidth. We believe more innovations in DSP techniques are needed to further reduce the cost per bit, increase network efficiency, and approach the Shannon limit. We anticipate that more optical functionalities will be achieved by DSP in the electrical domain, making future communication networks more efficient and flexible.

Acknowledgments: First of all, the guest editors would like to thank all the authors for their excellent contributions to this special issue. Secondly, we would like to thank all the reviewers for their outstanding job in evaluating the manuscripts and providing valuable comments. Additionally, the guest editors would like to thank the MDPI team involved in the preparation, editing, and managing of this special issue. Finally, we would like to express our sincere gratitude to Lucia Li, the contact editor of this special issue, for her kind, efficient, professional guidance and support through the whole process. It would not be possible to have the above collection of high quality papers without these joint efforts.

Conflicts of Interest: The authors declare no conflict of interest.

\section{References}

1. Soma, D.; Wakayama, Y.; Beppu, S.; Sumita, S.; Tsuritani, T.; Hayashi, T.; Nagashima, T.; Suzuki, M.; Yoshida, M.; Kasai, K.; et al. 10.16-Peta-B/s Dense SDM/WDM Transmission Over 6-Mode 19-Core Fiber Across the C+L Band. J. Lightwave Technol. 2018, 36, 1362-1368. [CrossRef]

2. Zhao, J.; Liu, Y.; Xu, T. Advanced DSP for coherent optical fiber communication. Appl. Sci. 2019, 9, 4192. [CrossRef]

3. Weng, Y.; Wang, J.; Pan, Z. Recent Advances in DSP Techniques for Mode Division Multiplexing Optical Networks with MIMO Equalization: A Review. Appl. Sci. 2019, 9, 1178. [CrossRef]

4. Zhou, H.; Li, Y.; Liu, Y.; Yue, L.; Gao, C.; Li, W.; Qiu, J.; Guo, H.; Hong, X.; Zuo, Y.; et al. Recent Advances in Equalization Technologies for Short-Reach Optical Links Based on PAM4 Modulation: A Review. Appl. Sci. 2019, 9, 2342. [CrossRef]

5. Yue, Y.; Wang, Q.; Anderson, J. Experimental Investigation of $400 \mathrm{~Gb} / \mathrm{s}$ Data Center Interconnect Using Unamplified High-Baud-Rate and High-Order QAM Single-Carrier Signal. Appl. Sci. 2019, 9, 2455. [CrossRef] 
6. Wang, Q.; Yue, Y.; Anderson, J. Compensation of Limited Bandwidth and Nonlinearity for Coherent Transponder. Appl. Sci. 2019, 9, 1758. [CrossRef]

7. Josten, A.; Baeuerle, B.; Bonjour, R.; Heni, W.; Leuthold, J. Optical Transmitters without Driver Amplifiers-Optimal Operation Conditions. Appl. Sci. 2018, 8, 1652. [CrossRef]

8. Jiang, T.; Zhao, L.; Liu, H.; Deng, D.; Luo, A.; Wei, Z.; Yang, X. Performance Improvement for Mixed RF-FSO Communication System by Adopting Hybrid Subcarrier Intensity Modulation. Appl. Sci. 2019, 9, 3724. [CrossRef]

9. Li, Y.; Ning, Q.; Yue, L.; Zhou, H.; Gao, C.; Liu, Y.; Qiu, J.; Li, W.; Hong, X.; Wu, J. Post-FEC Performance of Pilot-Aided Carrier Phase Estimation over Cycle Slip. Appl. Sci. 2019, 9, 2749. [CrossRef]

10. Shan, C.; Wu, X.-P.; Liu, Y.; Cai, J.; Luo, J.-Z. IBP Based Caching Strategy in D2D. Appl. Sci. 2019, 9, 2416. [CrossRef] 